\title{
ERRATUM
}

Fumihiko Kaneko · Hiroaki Yokomori - Aya Sato

Hajime Takeuchi · Kumiko Tahara - Yasunobu Sekiguchi

Shigehisa Mori • Tadashi Motoori • Hirobumi Kondo

Toshifumi Hibi

\section{A case of primary hepatic non-Hodgkin's lymphoma with chronic hepatitis C}

Med Mol Morphol (2008) 41:171-174

DOI 10.1007/s00795-008-0410-2

The legend for figure 3B appeared incorrectly in the article cited above. The correct legend is as follows.

Fig. 3B The diagnosis is non-Hodgkin's diffuse large B-cell lymphoma that is positive for CD20 (a), CD79a (b), B-cell lymphoma protein (bcl)-6 (d), and $\mathrm{Bcl} 2$ (e), but negative for $\mathrm{CD} 10$ (c) and multiple myeloma-1 (MUM-1) (f). a-f Immunohistochemical staining, $\times 600$

F. Kaneko $(\bowtie) \cdot$ H. Yokomori $\cdot$ A. Sato $\cdot$ H. Takeuchi $\cdot$ K. Tahara H. Kondo

Department of Internal Medicine, Kitasato Institute Medical Center

Hospital, 6-100 Arai, Kitamoto, Saitama 364-8501, Japan

e-mail: kaneko-f@insti.kitasato-u.ac.jp

T. Motoori

Department of Pathology, Kitasato Institute Medical Center

Hospital, Kitasato University, Kitamoto, Saitama, Japan

Y. Sekiguchi · S. Mori

Division of Hematology, Department of Internal Medicine, Saitama

Medical Center, Saitama Medical University, Kawagoe, Saitama,

Japan

T. Hibi

Department of Internal Medicine, School of Medicine, Keio

University, Tokyo, Japan 